\title{
Communication-based Collision Avoidance between Vulnerable Road Users and Cars
}

\author{
Michele Segata, Romas Vijeikis*, Renato Lo Cigno \\ Dept. of Information Engineering and Computer Science, University of Trento, Italy \\ romas.vijeikisestudenti.unitn.it, \{msegata, locigno\}@disi.unitn.it
}

\begin{abstract}
The advent of wireless communication for vehicles paves the road for a bounty of cooperative applications: The most interesting being cooperative safety awareness. By exchanging information vehicles can become aware of each other and prevent dangerous situations that can lead to crashes either by early warning drivers or by automatic vehicle control, a solution particularly appealing for self-driving cars. While research on vehicular safety mainly focuses on vehicle-to-vehicle safety, we can exploit communication to implement applications aimed at protecting vulnerable road users, such as pedestrians or cyclists In this paper, we start by exploiting a probability framework for estimating the likelihood of collision between a vehicles approaching an intersection and a cyclist, in light of the feasibility of communication between the two. On top of this framework, we design a simple application that, under certain conditions, informs the car driver (assumed to have to yield precedence) of the possible collision. We model the reaction of the driver to the warning and analyze possible benefits and drawbacks of such an application. The contribution is not the application itself, which is obvious, but the insights in the results in light of communication capabilities and human reaction that provide a specific set of aspects that should be considered in the design of such a safety system.
\end{abstract}

\section{INTRODUCTION}

Vehicular communication is fostering a wide range of research ideas that span from safety to efficiency, as well as comfort-oriented applications. Starting from the last category, we can list examples such as automatic toll collection, service announcements, and multimedia content download [1]; in practice, all the services that can improve the experience of drivers and passengers without a special focus to the vehicle itself. Efficiency applications, instead, focus on reducing travel times and fuel consumption, saving money and reducing carbon footprint of vehicles. Examples of these applications include optimal speed advisory systems for traffic lights [2], virtual traffic lights [3], and platooning [4]-[6]. Safety, however, is probably the main driver toward the development of cooperative vehicular applications, as there is a huge accident and death reduction potential.

The list of proposed vehicular safety applications is very large and includes traffic signal violation, cooperative forward collision warning, and emergency electronic brake lights to name a few [7], [8]. An important application, especially in urban environments, is intersection collision warning/assistance [9], [10]. The aim of the application is

\footnotetext{
${ }^{*}$ Romas Vijeikis work was supported by Movalia (https://www.movalia.it/) and EIT Digital (https://www.eitdigital.eu/).
}

to mitigate the effects or, if possible, completely avoid an accident at an intersection due to human mistakes or traffic violations. Its importance is fundamental, as the majority of urban accidents happen near or at an intersection.

Vehicular applications initially focused on car-to-car safety only, but there is no doubt that vulnerable road users such as pedestrians or cyclists are those who more often suffer fatal or serious injuries when involved in an accident: Car passengers are protected by the vehicle itself (chassis, safety belt, airbags), while pedestrian and cyclists are simply exposed. To have an idea of the problem, out of 5.9 million crashes in the European Union in 2013, 2.6 million involved pedestrians and cyclists [11].

For these reasons, in this paper we focus on an intersection collision avoidance application for cyclists. Inspired by the work in [12], which focuses on car-to-car collision avoidance, we analyze an intersection collision avoidance system aimed at protecting cyclists from vehicles that may collide with them. We adapt the concept of collision probability in [12] to model T-shaped intersections, that are very common in areas where a bike lane on a major urban road intersects a residential road. We model misbehaving car drivers that, while approaching the intersection, ignore a cyclist right-of-way due to, for example, a large object obstructing the line of sight. In this scenario, we analyze the collision rate without and with a warning application that exploits Bike-to-vehicle (B2V) communication to warn the driver about a possible collision. We model drivers' reaction time to the warning, investigating the benefits and drawbacks of such an application under different parameters, obtaining interesting and useful insights for a realworld implementation.

The main contribution of this paper is the analysis of the opportunity and timing of warning of the application in a scenario that takes into account most of the real constraints of a real scenario.

\section{BACKGROUND AND RELATED WORK}

Besides passive safety measures such as helmets, lightreflective clothes, and front and back lights that tries to avoid accidents or reduce their effects, we also find active safety systems installed in commercial cars. Some examples include autonomous emergency braking, which exploits a radar, a lidar, or a camera to detect objects in collision course and autonomously brake the car [13]. 


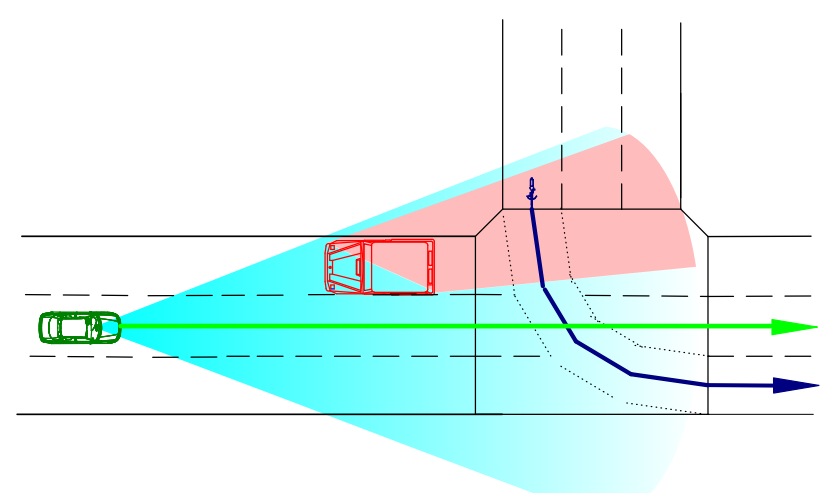

Figure 1: $\mathrm{T}$ intersection layout with blind zone problem.

While these systems can already reduce the risk of a collision or its consequences, they have an intrinsic limitation, which is their field of view. Sensor-based solutions become ineffective when there is no line of sight between the vehicle and the object in the collision course.

As an example, take the situation depicted in Figure 1. The picture represents a $\mathrm{T}$ intersection where the main road is the one going top to bottom and then turning to the right, indeed a left turn for a bike following the blue line trajectory. Vehicles coming from the left should give the right-of-way to bikes on the bike-lane of the main road. This layout is dangerous because the driver of a vehicle coming from the left might think of being on the main road just because it is straight, or simply being distracted by whatever reason. Such a mistake can be mitigated by sensor-based safety applications, but not when an object obstructs the line of sight: in this case sensors are no better than human eyes.

The alternative to pure sensor-based solutions is cooperation by means of communication. By actively exchanging data, vehicles and other road actors can get informed about each others' presence, preventively compute the risk of a collision, and, if needed, take countermeasures.

Classical Vehicle-to-vehicle (V2V) applications consider the use of an IEEE 802.11-based technology (i.e., IEEE $802.11 \mathrm{p} \mathrm{[14])} \mathrm{as} \mathrm{safety} \mathrm{mainly} \mathrm{requires} \mathrm{local} \mathrm{com-}$ munication. In addition, other technologies such as Visible Light Communication (VLC) [15] or 4G cellular (Long Term Evolution (LTE)) [16] have been proposed for the purpose. In this paper we consider classical V2V using IEEE 802.11p.

In the literature we find some bicycle safety-related projects that investigated the issue of vulnerable road users. One is the WATCH-OVER project [17], which focused on several aspects concerning the protection of vulnerable road users. The project investigated different scenarios, technologies, algorithms, and applications.

Another project is VRUITS [18]. This project also identified and investigated critical scenarios for vulnerable road users, showing that intersections are the most dangerous accident locations, especially in poor visibility conditions. In addition, the project took into account the human factor, e.g., developing intelligent traffic signals that take into account the time required to cross a street or an intersection.

BikeCOM [19], instead, focuses more on the development of communication strategies for cyclists and vehicles based on smartphones. The aim of the developed application was to establish a B2V connection between smartphones used in vehicles and by vulnerable road users to exchange safety related information.

Finally, we have the PROSPECT project [20]. The project is still ongoing and focuses on the development of active safety systems implementing independent emergency braking, focusing on better detection algorithms as well.

In this paper, rather than using an experimental approach, we focus on modeling. In particular, we model the misbehavior of vehicle drivers approaching an intersection as depicted in Figure 1. Position, speed and acceleration data are shared between cars and bikes using IEEE 802.11p, and the collision probability between a vehicle and a bicycle in a T-shaped intersection is computed extending [12]. Based on this probability, we define a threshold-based mechanism to inform the driver of the car about the incoming bicycle in collision course, simulating its reaction to the warning. We then study the impact of choosing different probability thresholds and distances to the intersection for issuing the warning.

\section{Protecting Vulnerable RoAd USERS}

\section{A. Computing a Collision Probability}

We extend the formulation of collision probability presented in [12] to include a T-shaped intersection. The whole formulation of the collision probability can be found in [12]. For our purposes it is sufficient to know that the concept of collision probability considers all possible trajectories that the the car and the bike can take while approaching the intersection exploiting the data shared via communication. The fraction of trajectories that led to a collision over all trajectories gives the probability of collision.

The computation considers the following parameters of two vehicles $A$ and $B$ approaching the intersection: speeds $\left(v_{A}, v_{B}\right)$, current distance to the intersection of the paths $\left(d_{A}, d_{B}\right)$, maximum acceleration and deceleration $\left(a_{A \max }\right.$, $\left.a_{A \min }, a_{B \max }, a_{B \min }\right)$ and current acceleration $\left(a_{A}, a_{B}\right)$. The distances $d_{A}$ and $d_{B}$ define the distance traveled by vehicles following their paths to the center point of intersection of their routes. These paths are not necessarily straight lines and depend on the topology on the roads leading to the junction. Based on these parameters, we can reconstruct all possible feasible trajectories and we compute the probability of collision based on how many of those overlap.

For the sake of completeness, we briefly list the formula for computing the probability of collision $\mathcal{P}_{c}$ :

$$
\mathcal{P}_{c}=\int_{a_{\min }}^{a_{\max }} p\left(a_{B}\right) \int_{a_{\min }}^{a_{\max }} p\left(a_{A}\right) \operatorname{coll}\left(\left[\begin{array}{l}
d_{A} \\
v_{A} \\
a_{A}
\end{array}\right],\left[\begin{array}{l}
d_{B} \\
v_{B} \\
a_{B}
\end{array}\right]\right) d a_{A} d a_{B}
$$


The formula, given the initial distance to the intersection and the speed of the vehicle, computes all possible trajectories obtained by choosing an acceleration in the range $\left[a_{\min }, a_{\max }\right]$ and check whether they would lead to a collision or not. In addition, the formula weights each single trajectory with the probability of choosing that specific acceleration. The computation can thus be customized by using different probability models.

To test for collision we check whether the two vehicles are inside the potential collision area at the same time, and this is done by computing the time at which a vehicle enters and leaves the collision area $\left(t_{e}\right.$ and $t_{l}$, respectively). The enter and leave times can be computed by considering the distances on the trajectory that correspond to the points where the vehicle enters and leaves the collision area (see Figure 2 for a graphical explanation). We can define these distances as $d_{e}$ and $d_{l}$, and, as originally done in [12] by solving the following equation

$$
d_{e / l}=\frac{a}{2} t_{e / l}^{2}+v t_{e / l}
$$

we can compute $t_{e}$ and $t_{l}$ as

$$
t_{e / l}= \begin{cases}-v+\frac{\sqrt{v^{2}+2 \cdot a \cdot d_{e / l}}}{a}, & \text { if } v^{2}+2 \cdot a \cdot d_{e / l} \geq 0 \\ \infty, & \text { otherwise }\end{cases}
$$

Equation (3), however, considers also cruising speeds which, especially for a bicycle, would be impossible. Differently to [12] we introduce a limit on the maximum speed reachable by vehicles and bikes. In particular we assume maximum speeds $v_{a}=72 \mathrm{~km} / \mathrm{h}$ and $v_{b}=50 \mathrm{~km} / \mathrm{h}$ for cars and bicycles, respectively. To account for maximum speeds we need to change Equation (3). First, we compute the time at which the vehicles reaches the maximum speed, i.e.,

$$
t_{v_{\max }}= \begin{cases}\frac{v_{\max }-v}{a}, & \text { if } a>0 \\ 0, & \text { otherwise }\end{cases}
$$

Then we compute the distance covered by the vehicle while reaching the maximum speed and the remaining distance to travel as

$$
d_{v_{\max }}=v \cdot \frac{a \cdot t_{v_{\max }}^{2}}{2}, \quad d_{\mathrm{after}}=d_{e / l}-d_{v_{\max }} .
$$

Finally, we can compute $t_{e}$ and $t_{l}$ accounting for maximum speed as

$$
t_{e / l}= \begin{cases}t_{e / l}(\text { Equation (3)), } & \text { if } t_{e / l} \leq t_{v_{\max }} \\ t_{v_{\max }}+\frac{d_{\mathrm{after}}}{v_{\max }}, & \text { otherwise. }\end{cases}
$$

The final point is defining the distribution for the probabilities $p\left(a_{A}\right)$ and $p\left(a_{B}\right)$ in Equation (1). In this work we draw the probability of acceleration from a triangular distribution having the minimum and maximum values set to $a_{\min }$ and $a_{\max }$, and the mode set to the current acceleration.

\section{B. Modeling Car Drivers'Behavior}

We consider the driver of the car to be the only actor in the scenario that can prevent the accident. The biker continues straight through the intersection because of the right of way

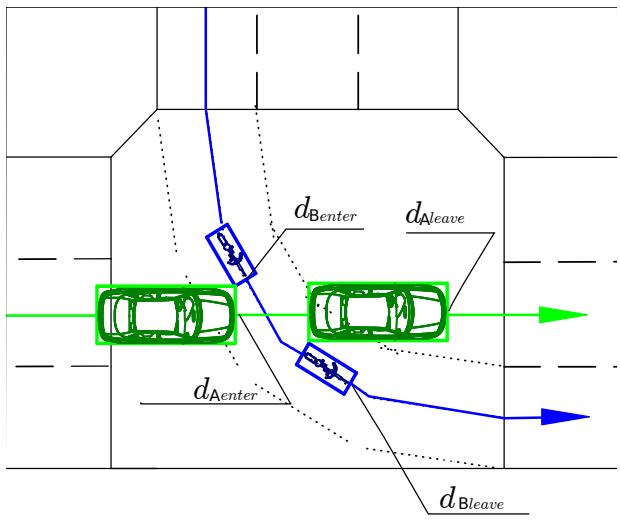

Figure 2: An example of identification of $d_{e}$ and $d_{l}$ positions for both vehicles on the intersection.

and it is the driver of the car, who receives a waning from the application and has to brake to avoid it.

We perform the analysis using the Veins vehicular networking framework [21], which couples the OMNeT++ network simulator with the SUMO vehicular mobility simulator. SUMO includes behavioral models that implement traffic rules, such as right of way, but it does not model "mistakes" and it is completely accident-free. To our purposes we need to modify the behavioral model, in this case the Intelligent Driver Model (IDM), to ignore traffic rules. This is done by disabling right of way rules when the vehicle is within a few meters to the intersection.

In addition, we model the reaction of the driver to warnings issued by the system. The bike periodically sends packets including its own position and speed. At each received update, the vehicle computes the collision probability $\mathcal{P}_{c}$ and informs the driver if $\mathcal{P}_{c}$ is above a certain threshold and if the vehicle is within a certain distance to the intersection. The rationale of the distance threshold is to limit the number of false positives, which can give the driver the impression that the application is not working. Both probability and distance thresholds are object of study in this paper.

When a warning is notified to the driver, the latter reacts by decelerating with maximum deceleration $a_{\min }$ down to a stop. We also consider a reaction time of $1 \mathrm{~s}$, so the driver does not immediately starts braking when receiving a warning.

\section{Performance Evaluation}

As anticipated in Section III-B we used the Veins vehicular networking simulation framework to analyze the performance of the proposed collision avoidance system in terms of safety. The simulation code is available upon request from the authors. First, we run a set of simulations where car drivers ignore the right of way and our collision avoidance application is disabled. This enables us to obtain collisions between vehicles and bicycles. In a second set of runs we enable our safety application measuring again the number of collisions.

In the simulation we inject pairs of cars and bicycles with randomized characteristics (Table I) to obtain a wide range of 


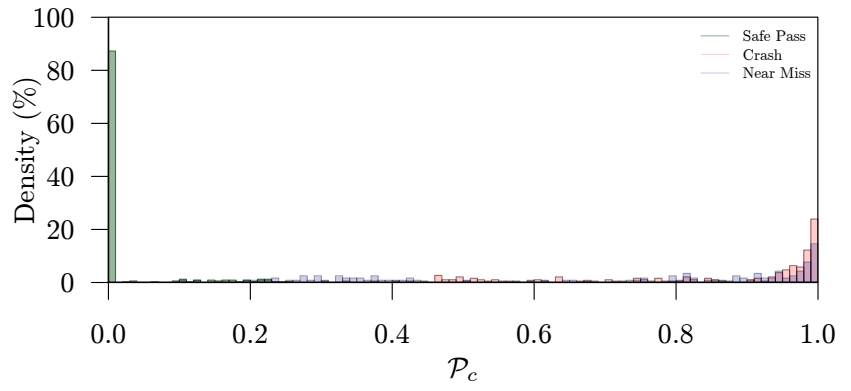

Figure 3: Distribution of computed collision probabilities $5 \mathrm{~m}$ from the intersection, safety system disabled.

situations, which include safe passes, near misses, and actual crashes. Near misses are a special kind of safe passes where the bike and the car get very close to each other. In this context, with "very close" we mean $2 \mathrm{~m}$.

With respect to communication, we consider an IEEE $802.11 \mathrm{p}$ network. Although it is not yet clear which kind of communication technology will support safety applications for vulnerable road users, we can assume this will be a local network available on user smartphones, such as Wi-Fi, LTE Direct, or 802.11p. In the context of the paper, however, the technology is irrelevant as our contribution is more application-oriented, and considering realistic communication as well as the effects of impairments is out of our scope. For the sake of completeness, Table II lists communication parameters used in the simulation.

Finally, we study the behavior of the system under different triggering conditions, in particular by choosing different collision probability thresholds and different minimum distances to the intersection. With respect to the probability threshold we consider the full range, i.e., from 0 to 1 , while for distances to the intersection we consider the range between $21 \mathrm{~m}$ and $14 \mathrm{~m}$, in steps of $1 \mathrm{~m}$. In total, we simulate 850 approaches per each set of parameters. We choose the parameters to intentionally design dangerous intersection approaches that can lead to a collision, a condition that is necessary for the evaluation of the system. Collision rates thus are not comparable to real-world situations.

Table I: IDM parameters for bicycles and cars used in the evaluation.

\begin{tabular}{ccc}
\hline Parameter & Cars & Bicycles \\
\hline$a_{\min }\left(\mathrm{m} / \mathrm{s}^{2}\right)$ & $\mathcal{U}(-6.5,-8.8)$ & $\mathcal{U}(-2.8,-3.5)$ \\
$a_{\max }\left(\mathrm{m} / \mathrm{s}^{2}\right)$ & $\mathcal{U}(2.8,3.5)$ & $\mathcal{U}(1.2,1.5)$ \\
$v_{\max }(\mathrm{m} / \mathrm{s})$ & $\mathcal{U}(8.5,17)$ & $\mathcal{U}(4.5,5.5)$ \\
$v(\mathrm{~m} / \mathrm{s})$ & $\mathcal{U}\left(0, v_{\max }\right)$ & $\mathcal{U}\left(0, v_{\max }\right)$ \\
\hline
\end{tabular}

\section{A. Collision Probability Overview}

The first step of our evaluation is reasoning on the behavior of the collision probability, easing the performance analysis of the application. Figure 3 shows the histogram of computed $\mathcal{P}_{c}$ values when the car is between $5 \mathrm{~m}$ and the center of the
Table II: Communication parameters.

\begin{tabular}{ll}
\hline Parameter & Value \\
\hline Path loss model & Free space $(\alpha=2.0)$ \\
Frequency & $5.89 \mathrm{GHz}$ \\
Bandwidth & $10 \mathrm{MHz}$ \\
Bit Rate & $18 \mathrm{Mbit} / \mathrm{s}$ \\
Transmit power & $20 \mathrm{~mW}$ \\
Noise floor & $-110 \mathrm{dBm}$ \\
Minimum sensitivity & $-89 \mathrm{dBm}$ \\
PHY model & IEEE $802.11 \mathrm{p}$ \\
MAC model & 1609.4 single channel $(\mathrm{CCH})$ \\
Beacon rate & $5 \mathrm{~Hz}$ \\
\hline
\end{tabular}

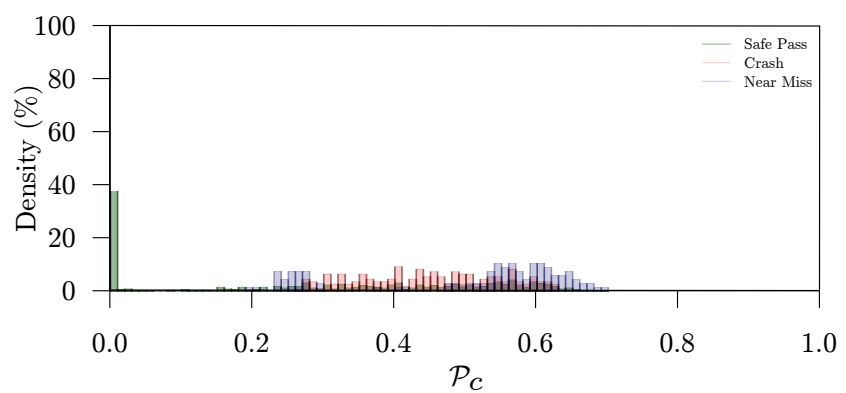

Figure 4: Distribution of computed collision probabilities $20 \mathrm{~m}$ from the intersection, safety system disabled.

intersection. In this scenario the collision avoidance assistance system is disabled.

The histogram shows a clear separation between probabilities computed during safe pass and crash scenarios. In particular, $\mathcal{P}_{c}$ for safe passes is always smaller than 0.25 , while crashes result in values of $\mathcal{P}_{c}$ larger than 0.5 , with some rare cases below 0.4 . The distribution of values for near misses is more "widespread", ranging from 0.1 to 1.0 . In the majority of the cases, however, near misses result in values of $\mathcal{P}_{c}$ larger than 0.9 .

This first observation shows that $\mathcal{P}_{c}$ correctly classifies intersection approaches, but it does not give a feeling about how effective it can be in reducing intersection collisions. In this first example $\mathcal{P}_{c}$ is computed $5 \mathrm{~m}$ to the intersection, where a collision might already be unavoidable. By taking the same measure $20 \mathrm{~m}$ from the intersection, however, the results change substantially (Figure 4). In particular, the range of $\mathcal{P}_{c}$ values shrinks, becoming no larger than 0.7 . In addition, values of $\mathcal{P}_{c}$ for safe passes and crashes overlap, indicating that using $\mathcal{P}_{c}$ as input for a collision avoidance system is not a trivial task, as we can easily produce false positives and false negatives.

Figure 5 explains the large difference between Figures 3 and 4 by plotting the evolution of $\mathcal{P}_{c}$ as function of the distance to the intersection for three intersection approaches, two of which led to a safe pass while the third to a crash. The plot shows that, $30 \mathrm{~m}$ from the intersection, distinguishing between the three situations is hardly possible, because of the large range of possible decisions that can still be made by the driver. For one safe pass (green dash-dotted line), $\mathcal{P}_{c}$ raises until $30 \mathrm{~m}$ to the intersection, where it finally drops to $020 \mathrm{~m}$ to the intersection, correctly identifying the absence of risk. For the 


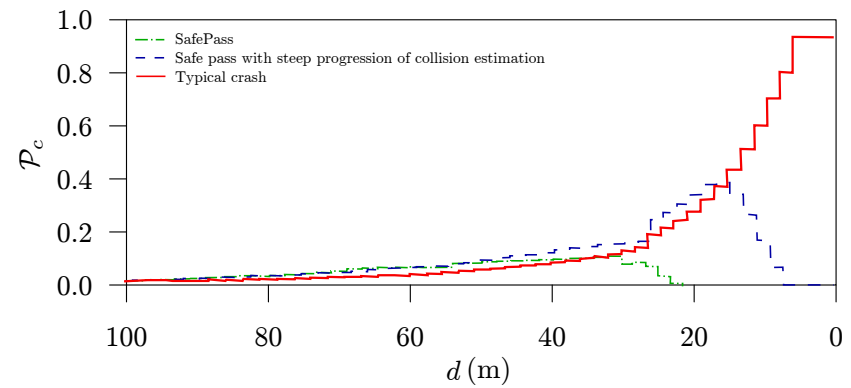

Figure 5: Evolution of the collision probability for three different intersection approaches.

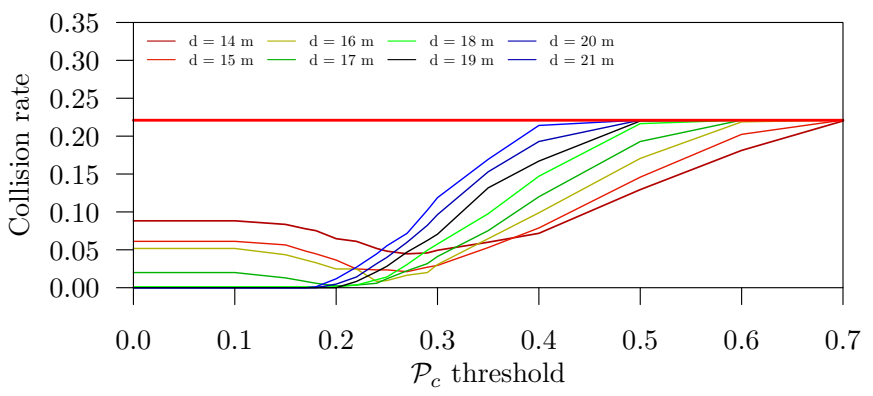

Figure 6: Collision rates as function of different $\mathcal{P}_{c}$ thresholds and different minimum distances to the intersection.

second safe pass (blue dashed line) $\mathcal{P}_{c}$ is still comparable to the crash approach up until roughly $20 \mathrm{~m}$ to the intersection, where it starts dropping to 0 . The crash approach correctly computes a $\mathcal{P}_{c}$ value of 1 , but only in the last 10 to $5 \mathrm{~m}$.

Figure 5 thus shows that the design of such an application is non trivial, and can easily lead to misjudgement, and thus false positives or false negatives.

\section{B. Intersection Collision Safety Evaluation}

The choice for investigating warning notifications distances between $21 \mathrm{~m}$ and $14 \mathrm{~m}$ is motivated by the results in Section IV-A. In this range, indeed, the computed collision probability becomes meaningful and can be exploited by the safety system. In this section we thus study the reduction in collision rates, as well as the balance between false positives and false negatives. The reader should bear in mind that the results in this section do not represent the ground truth of what is achievable by an intersection collision avoidance system and that they depend on our specific implementation and modeling. The results represent food for thoughts for the development of this kind of systems.

Figure 6 shows the collision rate as function of different $\mathcal{P}_{c}$ threshold and minimum distances to the intersection. For the sake of comparison, we also plot the collision rate for the approaches where the assistance application is disabled, which, in this particular setup, is around $22 \%$. For large minimum distances (between $21 \mathrm{~m}$ and $18 \mathrm{~m}$ ) and low $\mathcal{P}_{c}$ thresholds (up to 0.2), the application results in no crashes at all. As

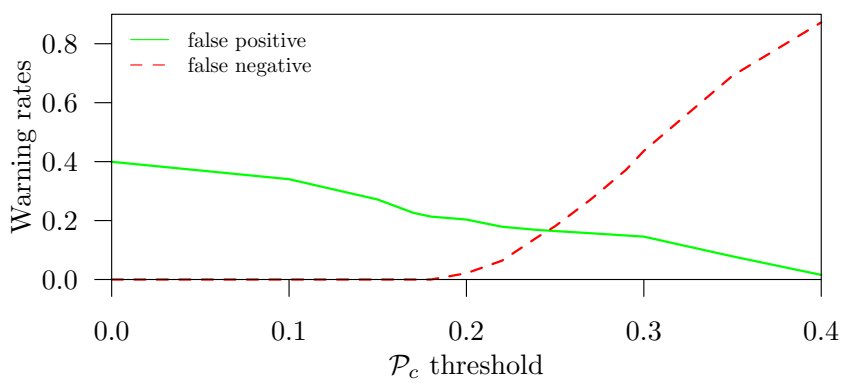

Figure 7: Comparison between false positives and false negatives as function of different $\mathcal{P}_{c}$ thresholds, for a minimum warning distance of $20 \mathrm{~m}$.

we increase the $\mathcal{P}_{c}$ threshold above 0.2 , the application starts to loose performance approaching the no-assistance collision rate for a $\mathcal{P}_{c}$ threshold that depends on the minimum warning distance.

For shorter minimum warning distances, instead, the behavior changes. First we see that the collision rate for a $\mathcal{P}_{c}$ threshold of 0 is not null, and the reason lies behind the fact that the vehicle might not have enough braking distance to stop or avoid the biker. In fact, as we increase the $\mathcal{P}_{c}$ threshold, the collision rate decreases before converging to the no-assistance collision rate While this might seem non intuitive, the explanation is fairly simple. In some cases, by informing the driver we actually cause a collision, which would not happen otherwise because the car would have crossed the intersection before the bike.

This shows a fundamental issue of these safety systems, that is when and if to inform a driver at all. The graph suggests that the vehicle should never be in a situation where nothing can be done anymore, or where the situation can be made worse. The system should not be responsible for accidents, but it should only avoid or mitigate them. This suggests that warnings should not be triggered in such cases, but this statement is very difficult to judge from a social and legal point of view. In addition, here we do not consider the impact force, which could also be an interesting metric of evaluation. In any case this aspect must be accounted for in the design of safety systems.

The final question regarding the results in Figure 6 is the cost of having no crashes. For large minimum warning distances and low $\mathcal{P}_{c}$ thresholds we completely avoid crashes, but, as Figure 7 shows, the rate of false positives is high. The Figure also shows that instead false negatives can be reduced to zero maintaining the threshold $\mathcal{P}_{c}$ large enough. To evaluate false negatives, we compute the amount of approaches that led to a crash or a near miss but where the warning was not issued to the driver. The graph shows a high false positives rate, which is clearly an undesired feature in terms of marketing, as users experiencing large false positives rates might think the system does not properly work and disable it. However, we have to keep in mind that these results have been obtained simulating only dangerous situations. Indeed, if we consider a rate of safe to dangerous situation of 100 to 1 (very reasonable, otherwise 
accidents would happen by the day to every one of us) and a $20 \%$ of false positives, this means that there is one false positive every 500 times a car intersects with a bike on a $\mathrm{T}$ shaped intersection, which is probably acceptable to users.

Depending on design goals, the $\mathcal{P}_{c}$ threshold can be tuned accordingly. We can choose to minimize the false positives rate while having no collisions at all, minimize the combination of false positives and false negatives, or minimize the false positives rate while keeping false negatives within an acceptable bound. For example, in this particular case setting a $\mathcal{P}_{c}$ threshold of 0.3 maintains the false positives rate below $20 \%$ and the collision rate (Figure 6) around $10 \%$ halving the number of collisions.

In conclusion, this evaluation highlights challenges and potentials of collision assistance systems showing that, during the design phase, we should also take into account social and legal aspects, and not only focusing on maximizing the safety gain.

\section{Concluding Discussion And Future Work}

This work presented a preliminary study on the applicability of vehicular communication for protecting vulnerable road users through an intersection collision avoidance application. We adapted the concept of collision probability in [12] to a T-shaped intersection and exploited it to issue collision warning to a driver. We modeled the misbehavior of a car driver and his/her reaction to a warning issued by the assistance system to discover some fundamental properties that should be accounted for in the design phase.

We showed that there are some fundamental issues to be addressed. In particular, we need to carefully choose the conditions for triggering a warning, in terms of collision probability and distance to the intersection. A low collision probability threshold can drastically reduce the collision rate, but at the cost of high false positives rate. In addition, the safety application should inform the driving within a reasonable distance to the intersection, so that there is still the possibility of avoiding a collision.

The next steps include a more realistic modeling of both car drivers and cyclists. Simulation is an effective mean to study the impact of these applications on the safety of road users, but the results must be built upon realistic and credible models. This can be done for instance by considering reconstructions of real world accidents (to model misbehaving drivers) and by experimenting the human reaction to warnings while driving. Improving the model with real data we can disregard the trajectories that are improbable, improving the estimation of the collision likelihood.

On top of the models and the best practices presented in this work we can start designing effective applications aimed at improving the safety of vulnerable road users.

\section{REFERENCES}

[1] T. K. Mak, K. P. Laberteaux, and R. Sengupta, "A Multi-channel VANET Providing Concurrent Safety and Commercial Services," in 2nd ACM International Workshop on Vehicular Ad Hoc Networks (VANET 2005). Cologne, Germany: ACM, September 2005, pp. 1-9.
[2] D. Eckhoff, B. Halmos, and R. German, "Potentials and Limitations of Green Light Optimal Speed Advisory Systems," in 5th IEEE Vehicular Networking Conference (VNC 2013). Boston, MA: IEEE, December 2013, pp. 103-110.

[3] T. Neudecker, N. An, O. K. Tonguz, T. Gaugel, and J. Mittag, "Feasibility of Virtual Traffic Lights in Non-Line-of-Sight Environments," in 9th ACM International Workshop on Vehicular Internetworking (VANET 2012). Low Wood Bay, Lake District, UK: ACM, June 2012, pp. 103-106.

[4] C. Bergenhem, Q. Huang, A. Benmimoun, and T. Robinson, "Challenges of Platooning on Public Motorways," in 17th World Congress on Intelligent Transport Systems (ITS 2010), Busan, Korea, Oct. 2010.

[5] S. Santini, A. Salvi, A. S. Valente, A. Pescapè, M. Segata, and R. Lo Cigno, "A Consensus-based Approach for Platooning with InterVehicular Communications," in 34th IEEE Conference on Computer Communications (INFOCOM 2015). Hong Kong, China: IEEE, Apr. 2015, pp. 1158-1166.

[6] M. Segata, "Safe and Efficient Communication Protocols for Platooning Control," PhD Thesis (Dissertation), University of Innsbruck, February 2016.

[7] National Highway Traffic Safety Administration, "Vehicle Safety Communications Project Task 3 Final Report: Identify Intelligent Vehicle Safety Applications Enabled by DSRC," NHTSA, Project Report DOT HS 809 859, March 2005.

[8] M. Segata and R. Lo Cigno, "Automatic Emergency Braking - Realistic Analysis of Car Dynamics and Network Performance," IEEE Transactions on Vehicular Technology, vol. 62, no. 9, pp. 4150-4161, Oct. 2013.

[9] L. Le, A. Festag, R. Baldessari, and W. Zhang, "Vehicular Wireless Short-Range Communication for Improving Intersection Safety," IEEE Communications Magazine, vol. 47, no. 11, pp. 104-110, November 2009.

[10] S. Joerer, B. Bloessl, M. Segata, C. Sommer, R. Lo Cigno, A. Jamalipour, and F. Dressler, "Enabling Situation Awareness at Intersections for IVC Congestion Control Mechanisms," IEEE Transactions on Mobile Computing, vol. 15, no. 7, pp. 1674-1685, June 2016.

[11] "Road Safety in the European Union: Trends, Statistics and Main Challenges," European Union, Report MI-AC-15-001-EN-N, March 2015.

[12] S. Joerer, M. Segata, B. Bloessl, R. Lo Cigno, C. Sommer, and F. Dressler, "A Vehicular Networking Perspective on Estimating Vehicle Collision Probability at Intersections," IEEE Transactions on Vehicular Technology, vol. 63, no. 4, pp. 1802-1812, May 2014.

[13] D. Hynd, M. McCarthy, J. Carroll, M. Seidl, M. Edwards, C. Visvikis, M. Tress, N. Reed, and A. Stevens, "Benefit and Feasibility of a Range of new Technologies and Unregulated Measures in the Field of Vehicle Occupant Safety and Protection of Vulnerable Road Users," European Union, Report NB-07-14-108-EN-N, March 2015.

[14] IEEE, "Wireless Access in Vehicular Environments," IEEE, Std 802.11p2010, Jul. 2010.

[15] M. Segata, R. Lo Cigno, H.-M. Tsai, and F. Dressler, "On Platooning Control using IEEE $802.11 \mathrm{p}$ in Conjunction with Visible Light Communications," in 12th IEEE/IFIP Conference on Wireless On demand Network Systems and Services (WONS 2016). Cortina d'Ampezzo, Italy: IEEE, January 2016, pp. 124-127.

[16] S. Kato, M. Hiltunen, K. Joshi, and R. Schlichting, "Enabling Vehicular Safety Applications over LTE Networks," in 2nd International Conference on Connected Vehicles \& Expo (ICCVE 2013), Las Vegas, NV, USA, December 2013, pp. 747-752.

[17] L. Andreone and G. Wanielik, "Vulnerable Road Users Thoroughly Addressed in Accident Prevention: The WATCH-OVER European Project," in 14th World Congress on Intelligent Transport Systems (ITS 2007). Beijing, China: ITS Japan, October 2007, pp. 629-636.

[18] J. Scholliers, D. Bell, A. Morris, and A. B. García, "Improving Safety and Mobility of Vulnerable Road Users through ITS Applications," in 5th Transport Research Arena (TRA 2014). Paris, France: IFSTTAR, April 2014.

[19] P. Gustafsson, J. Muñez, L. Lindgren, C. Boda, and M. Dozza, "BikeCOM: Cooperative Safety Application Supporting Cyclists and Drivers at Intersections," in 3rd International Conference on Driver Distraction and Inattention, Gothenburg, Sweden, September 2013.

[20] I. Gohl, A. Schneider, J. Stoll, M. Wisch, and V. Nitsch, "Car-to-cyclist Accidents from the Car Driver's Point of View," in 5th International Cycling Safety Conference (ICSC2016), Bologna, Italy, November 2016.

[21] C. Sommer, R. German, and F. Dressler, "Bidirectionally Coupled Network and Road Traffic Simulation for Improved IVC Analysis," IEEE Transactions on Mobile Computing, vol. 10, no. 1, pp. 3-15, Jan. 2011. 